\title{
POSSIBILITIES TO REDUCE POLLUTANT EMISSIONS IN NAVAL DIESEL ENGINES
}

\author{
Mihail Lucian DUMITRACHE ${ }^{1)}$, Catalin FAITAR ${ }^{1 *}$ \\ ${ }^{1)}$ Maritime University of Constanta, Faculty of Naval Electromechanics, 104 Mircea cel Batran, \\ RO-900663, Constanta, Romania \\ * Corresponding author: catalinfaitar@yahoo.ro
}

\begin{abstract}
The combustion process is, by far, the most important and complex process that takes place in engines. Its importance is given by the fact that it provides the flow of energy used in the engine and is the source of all pollutant emissions, the efficiency of the engine being directly influenced by it. The mechanisms of combustion are particularly complex and are not fully known even today, the most difficult problem being the mechanisms of mixture formation and the chemistry of the combustion process. Over time, depending on the evolution of knowledge in the field and computer technology, various mathematical models have been developed, which have. Emission estimation and theoretical verification, in the first phase, of the solutions applicable to in-service engines could greatly reduce research and production costs, given that there are a variety of engines on board ships and measurements in operation are very difficult.
\end{abstract}

Keywords: stand, NOx, engine, simulation

\section{INTRODUCTION}

The combustion process is by far the most important and complex process that takes place in engines, its importance being derived from the supply of energy flow used in the engine, respectively is the source of all pollutant emissions, engine efficiency is directly influenced by it.

The engine chosen for the investigations is D110 (naval landed T650 engine), frequently encountered in the port-river maritime field (as Diesel-generator), an area for which a stricter application of the regulations of the Protocol is foreseen.

The developed model is of multidimensional type, solving systems of specific equations: a. compressible turbulent flow; b. chemical reactions (combustion of fuel, respectively noxious emissions); c. the flow and evaporation of jets of liquid particles. The formulation is spatially two-dimensional and allows the plane and axially symmetrical approach of the combustion chamber geometry (facilitates the consideration of the swirl motion around the axis of symmetry, increasing the spatial resolution and partially implementing the third geometric dimension). The numerical solving scheme is ahead of time, using a finite volume discretization and a partial default algorithm. The discretization network is adjustable and consists of generalized quadrilaterals whose corners are specified by timedependent coordinates relative to the lower position of the piston, which allows the Eulerian or Lagrangean problem to be approached as needed.

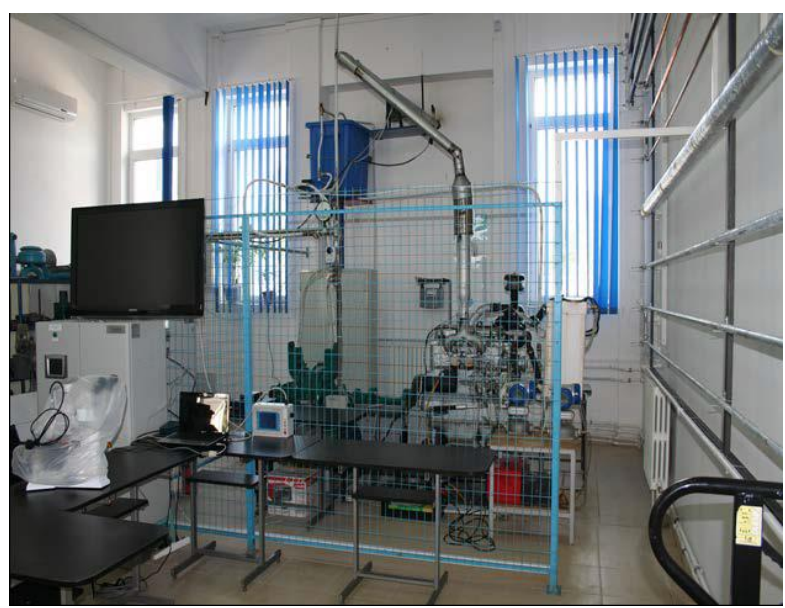

Fig. 1. T650 engine test stand

In Figure 1, the test stand was prepared in accordance with the recommendations of ISO/TC 70/SC8 of 21.09.1995, the 1997 Protocol on the amendment of MARPOL 73/78 and Directive 97/68/EC of 16.12.1997, both in terms of 
composition and construction, as well as the accuracy of the equipment and the type of measurement methods used. The engine testing stand, existing in the Multifunctional Laboratory at the Nautical Base of the Maritime University, is intended for testing engines with internal combustion with power below $100 \mathrm{hp}$, speed below $5000 \mathrm{rpm}$ and maximum torque at shaft below $300 \mathrm{Nm}$. It was configured, in stages, through its own financial efforts, being purchased aggregates from several suppliers (Uzina Tractorul Brașov, KROHNE, Siemens etc.), developed complex dedicated systems with specialized companies (Syscom 18 SRL, ECO Electroservice SRL) and self-directed works [1].

\section{CALCULATION OF THE INJECTION FEED RATE CHARACTERISTIC FOR THE T650 ENGINE}

The application - aimed at verifying the optimal range for the injection advance, so as to minimize NOx emissions.

Among the possibilities to reduce NOx emissions, the change in the injection advance is the most important, because: a. NOx emissions are strongly influenced by the size of the injection advance; $b$. injection advance modification is most convenient for in-service engines, because it is easy to execute.

The used input data and the results obtained are in Table 1.

Table 1. Results

\begin{tabular}{|c|c|c|c|c|c|c|}
\hline Speed & $\begin{array}{c}\begin{array}{c}\text { Real } \\
\text { advance }\end{array} \\
\end{array}$ & NOx & NOx-calc & Error & Consumption & Power \\
\hline [rpm] & [deg] & [ppm] & [ppm] & {$[\%]$} & [g/kWh] & {$[\mathbf{k W}]$} \\
\hline \multirow{7}{*}{2400} & 0.2 & 752 & 776 & - & 258.2074 & 47.64 \\
\hline & 1.3 & 782 & 858 & -9.718 & 256.1854 & 48.34 \\
\hline & 2.2 & 850 & 929 & -9.294 & 250.0907 & 49.59 \\
\hline & 3.7 & 964 & 1056 & -9.543 & 244.2857 & 50.4 \\
\hline & 4.2 & 1008 & 1101 & -9.226 & 242.8684 & 50.62 \\
\hline & 5.4 & 1112 & 1215 & -9.262 & 241.5013 & 50.89 \\
\hline & 6.5 & 1211 & 1327 & -9.578 & 239.3538 & 51.38 \\
\hline \multirow{7}{*}{2000} & 0.4 & 840 & 876 & -4.285 & 246.2234 & 43.16 \\
\hline & 1.1 & 881 & 932 & -5.788 & 244.9954 & 43.76 \\
\hline & 2.5 & 952 & 1067 & -12.07 & 238.7025 & 44.7 \\
\hline & 3.7 & 1130 & 1234 & -9.203 & 235.0066 & 45.42 \\
\hline & 4.2 & 1177 & 1303 & -10.70 & 233.7349 & 45.65 \\
\hline & 5.2 & 1321 & 1446 & -9.462 & 230.276 & 46.01 \\
\hline & 6.4 & 1450 & 1621 & -11.793 & 229.2646 & 46.37 \\
\hline \multirow{7}{*}{1600} & 1.7 & 1074 & 1176 & 9.497 & 233.4771 & 37.1 \\
\hline & 2.5 & 1136 & 1258 & -10.739 & 233.4846 & 37.45 \\
\hline & 3.7 & 1266 & 1405 & -10.979 & 229.1426 & 38.02 \\
\hline & 4.9 & 1435 & 1564 & -8.990 & 226.762 & 38.45 \\
\hline & 5.7 & 1471 & 1649 & -12.101 & 226.0509 & 38.54 \\
\hline & 6.9 & 1631 & 1797 & -10.178 & 223.8922 & 38.59 \\
\hline & 7.6 & 1752 & 1897 & -8.276 & 223.4944 & 38.69 \\
\hline \multirow{7}{*}{1440} & 2.6 & 1156 & 1232 & -6.574 & 231.1098 & 33.88 \\
\hline & 3.5 & 1221 & 1321 & -8.190 & 232.5629 & 34.18 \\
\hline & 4.6 & 1344 & 1455 & -8.259 & 228.125 & 34.56 \\
\hline & 5.6 & 1498 & 1573 & -5.007 & 226.1494 & 34.8 \\
\hline & 5.9 & 1557 & 1612 & -3.532 & 225.7233 & 34.91 \\
\hline & 7.3 & 1689 & 1784 & -5.625 & 224.5634 & 34.93 \\
\hline & 8.3 & 1792 & 1904 & -6.250 & 224.7998 & 34.96 \\
\hline \multirow{7}{*}{1100} & 2.8 & 1377 & 1546 & -12.273 & 229.8737 & 25.34 \\
\hline & 3.8 & 1470 & 1672 & -13.741 & 229.4442 & 25.37 \\
\hline & 4.3 & 1595 & 1749 & -9.655 & 227.212 & 25.43 \\
\hline & 5.7 & 1764 & 1927 & -9.240 & 226.7661 & 25.48 \\
\hline & 6.1 & 1803 & 1967 & -9.096 & 227.4281 & 25.74 \\
\hline & 7.3 & 1921 & 2103 & -9.474 & 227.7085 & 25.66 \\
\hline & 8.6 & 2029 & 2261 & -11.434 & 228.2362 & 25.57 \\
\hline
\end{tabular}




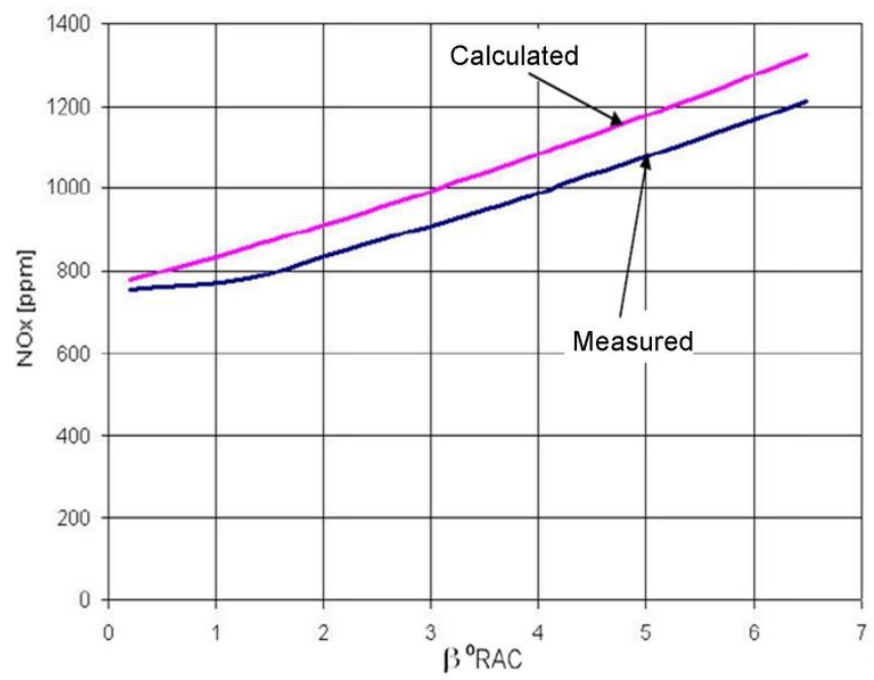

Fig. 2. NOx variation at $2400 \mathrm{rpm}$ and $100 \%$ load

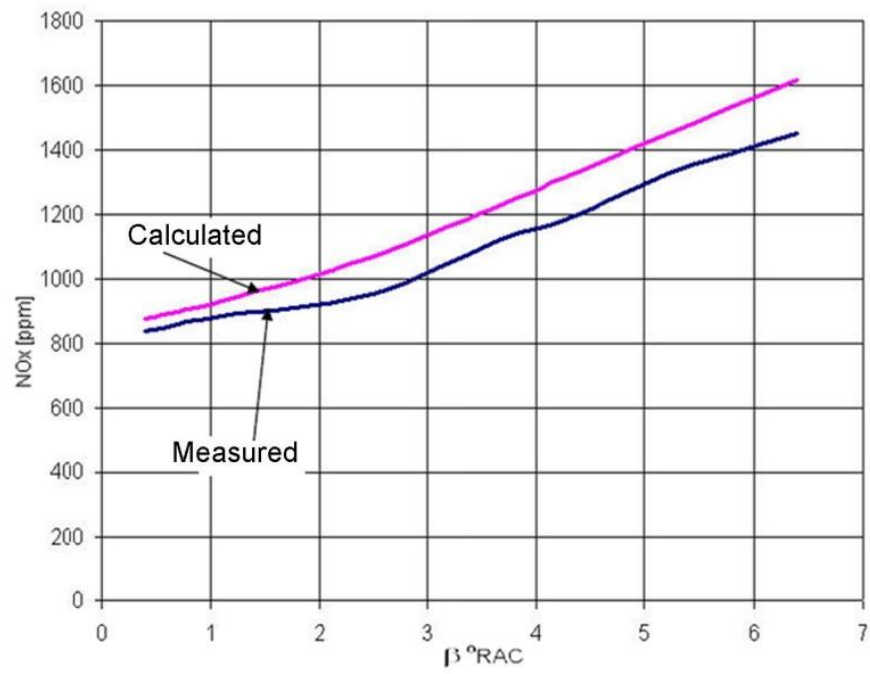

Fig. 3. NOx variation at $2000 \mathrm{rpm}$ and $100 \%$ load

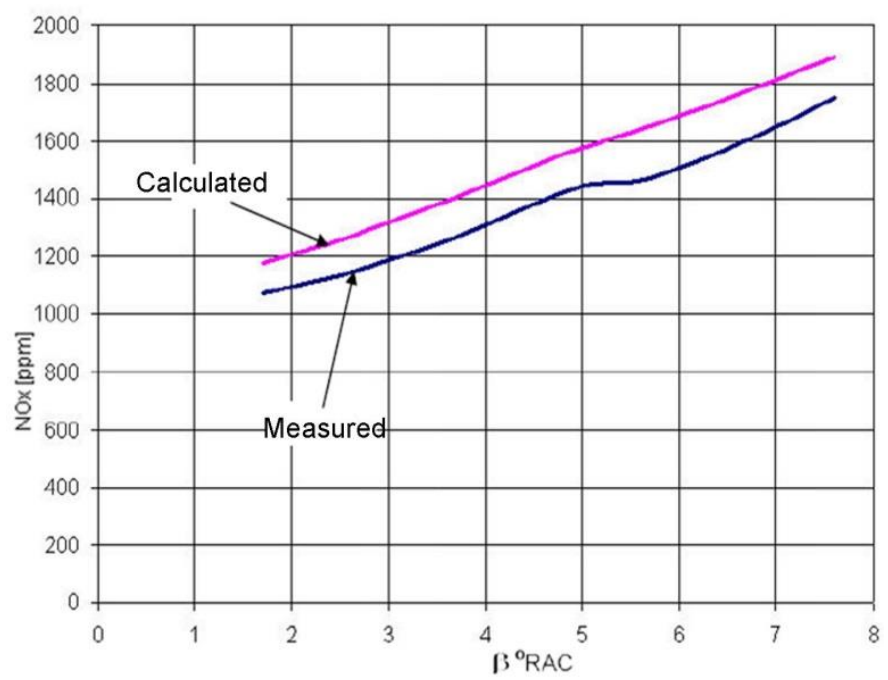

Fig. 4. NOx variation at $1600 \mathrm{rpm}$ and $100 \%$ load 


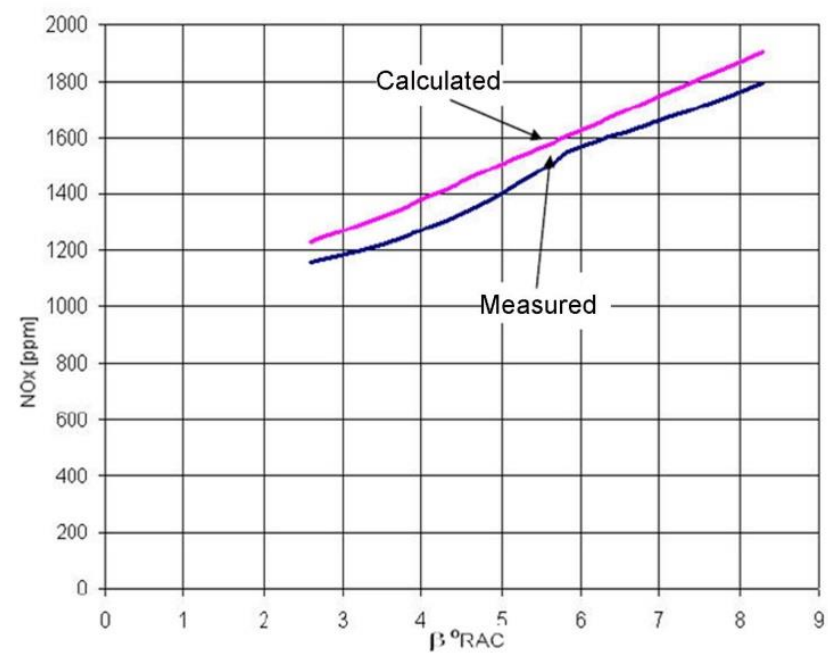

Fig. 5. NOx variation at $1440 \mathrm{rpm}$ and $100 \%$ load

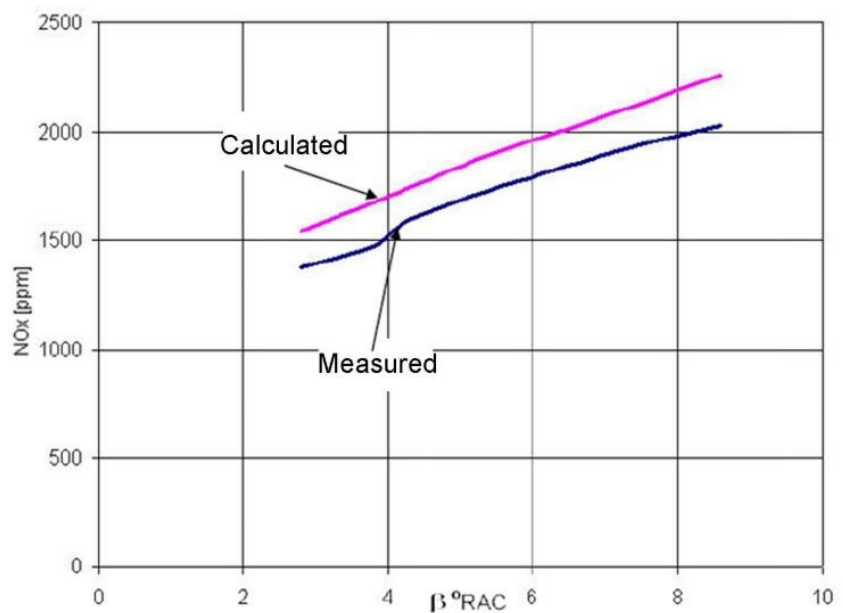

Fig. 6. NOx variation at $1100 \mathrm{rpm}$ and $100 \%$ load

The error between the measured and calculated values is between 3 and $12 \%$, most being in the range of $8-10 \%$, being relatively large, but satisfactory, taking into account the complexity of the phenomenon and the relatively low capabilities of the model. At partial loads the errors can be higher. The program can be used to study the influence of injection advance on NOx emissions. It can be seen that the results are better for the regimes at which the calibration was performed (speeds of $2400 \mathrm{rpm}$ and $1440 \mathrm{rpm})$ [2].

In line with the established objectives, the study of the possibilities to reduce NOx emissions for in service engines is particularly important. Of these, changing the injection advance is the most important because:

- it is well known that NOx emissions, like the whole combustion process, are strongly influenced by the size of the injection advance;

- the modification of the injection advance is the most convenient modification that can be made to some engines in operation, because it is easy to execute, does not involve additional costs and does not remove the motors from an operation, the adjustment can be made during a periodic overhaul.

The calculations were performed for the cases, which contain data on maximum load regimes, in which, as shown above, it is expected that the results will be as close as possible to reality and we can highlight the influence advance to injection.

The model was calibrated as in the previous cases so that no adjustments were required after each case and, in addition, the values chosen were those in the literature not optimized for the specific case. In this way, the aim was to obtain the highest possible degree of generality.

The input data used and the results obtained from the calculation are presented in table 1 for five operating speeds [3], [4].

\section{INTERPRETATION OF RESULTS}

Analyzing the calculated errors presented in table 1 , it is found that the error between the measured and calculated values is between 3 and $12 \%$, most being in the range of $8-10 \%$. These values 
were somewhat expected given the results obtained in the other cases.

The error obtained is relatively large, but given the complexity of the phenomenon and the relatively low capabilities of the model, it can be considered satisfactory mainly due to a favourable distribution. It can be seen that the error is:

- permanently unilateral, in the sense that the calculated value is always higher than the measured value;

- the dispersion of errors for each case is less than $5 \%$;

- the correlation between the evolution trends of the two quantities is observed and is good, the linear dependence between the NOx emission concentrations and the advance is known.

As measurements and calculations were performed only on the external characteristic for each injection feed adjustment, it is expected that the errors will be higher at partial loads. The calculation program, as presented in previous cases, has more modest performance when calculating partial loads.

The quasi-linear dependence between NOx emissions and injection advance as can be seen in figures 2 - 6 is well known in the literature.

The conclusion is that the program can be used to study the influence of injection advance on NOx emissions, provided that the results are viewed with discernment. It can be easily seen that the results are better for the regimes at which the calibration was performed (speeds of $2400 \mathrm{rpm}$ and $1440 \mathrm{rpm}$ ) [5], [6].

\section{CONCLUSIONS}

The objective of this scientific article was to develop a calculation program for estimating the pollutant emissions produced by naval diesel engines, in particular NOx emissions regulated by Annex 6 of the 1997 Protocol [8], [9], which entered into force in 2010. The proposed objective has been achieved, but its applicability remained only in the sphere of science and research. Originally conceived as a tool for assessing the impact of Annex VI on Romanianflagged ships, it has become uninteresting for parties involved in decision-making, due to the dramatic decrease in the number of ships.

Thus, it is currently made in open architecture, in the form of a collection of subroutines and independent functions that implement the many mathematical models used, called from a main program framework. This program allows for easy entry of initial data, manipulation and storage, as well a display of final results without benefiting from an overly elaborated user interface. It was designed in a modular way, so that it could be easily modified, enriched and perfected later. This, however, makes it difficult for an untrained and inexperienced operator to use.
The presented method was dedicated to the study of the possibilities to improve the quality of the results, especially those related to NOx emissions, which was materialized in the application of the work. Two new modules have been introduced for the calculation of NOx in flame and from nitrogen bound in fuel. The resulting conclusions were:

- in the proposed form, they do not significantly improve the obtained results,

- a key issue remains the fuel combustion mechanism, which is far too complex to be modelled to meet all the requirements of proper simulation and the accuracy of results and ease of solving [7].

\section{REFERENCES}

[1] Sabău A., Buzbuchi N., Sabie S. (2003) Modelarea jetului axisimetric, Analele Universităţii Maritime din Constanța, Constanţa.

[2] Sabău A., Dumitrache C. (1999) Modelarea procesului de ardere în motoarele Diesel folosind metoda ratei de combustibil, Analele Univerisităţii "Ovidius", 1999.

[3] Dumitrache C., Sabău A., Comandar C., Amariei N. (2002) The computation of steel hardening cycle, pp. 193-196, Analele Universității Maritime, Constanța.

[4] Buzbuchi N., Sabău A., Şoloiu V.A. (2001) Marine I.C.E. Thermodynamics Processes Modelling and Computer Simulation, the 11th National Conference on Thermodynamics, Galaţi.

[5] Bayhan M., Buzbuchi N. (1993) Development and application of marine engine, Sesiunea de comunicări ştiinţifice, Universitatea "Dunărea de Jos ", Galaţi.

[6] *** A motorship supplement ship repair, March 1993.

[7] Cebeci T. (1987) Analysis of Turbulent Boundary Layers, Pergamon Press.

[8] *** The Protocol of 1997 (MARPOL Annex VI) MARPOL 73/78 Annex VI - Regulations for the prevention of Air Pollution from ships - Technical and Operational implications, Det Norske Veritas, Available: http://hulpinnood.nl/wpcontent/uploads/2015/03/BIJLAGE3_MarpolannexVI.pdf (March 2018)

[9] Čampara L., Hasanspahić N., Vujičić S., (2018) Overview of MARPOL ANNEX VI regulations for prevention of air pollution from marine diesel engines, GLOBMAR 2018SHS Web of Conferences 58 , 01004

https://doi.org/10.1051/shsconf/20185801004,. 